\title{
Conduction Activation Energy in PEDOT:PSS Thin Films
}

\author{
Tahereh Akbari, Benyamin Harkinezhad, Faramarz Hossein-Babaei \\ Electronic Materials Laboratory, Electrical Engineering Department, K. N. Toosi University of \\ Technology, Tehran, 16317-14191, Iran \\ fhbabaei@kntu.ac.ir
}

\begin{abstract}
:
Conductive polymer PEDOT:PSS has been used for the fabrication of humidity and VOC sensors. The electrical conduction mechanism in this polymer is established, but the reported conduction activation energy $\left(E_{a}\right)$ values vary in a wide range. Here, we report measuring $E_{a}$ in different PEDOT:PSS samples and show that, indeed, $E_{a}$ varies with the sample thickness and the relative humidity $(\mathrm{RH})$ in the surrounding atmosphere. The results are explained by the assumption of multiple carrier excitation mechanisms. Three devices with different resistances at room temperature are constructed and the change in the device resistance is measured at different temperatures in environments with different $\mathrm{RH}$ levels. The results, surprisingly, indicates almost a linear relationship between the $\mathrm{E}_{\mathrm{a}}$ and the RH; for every $5 \%$ increase in the $\mathrm{RH}, \mathrm{E}_{\mathrm{a}}$ increases by approximately $7 \%$.
\end{abstract}

Key words: PEDOT:PSS, Relative humidity, electrical conduction mechanism, activation energy

\section{Introduction}

The excellent physical and chemical properties of conducting polymers make them attractive materials for electronic applications [1, 2]. One of the wellknown and widely used conducting polymers is poly(3,4-ethylenedioxythiophene):polystyrene

sulphonate (PEDOT:PSS). The mechanism of charge transport in PEDOT:PSS films however, is yet to be fully understood. The most commonly accepted model is the variable range hopping (VRH) model, as proposed by Aleshin et al. [3]. Here, we investigate the effect of thickness and relative humidity on the conduction activation energy of PEDOT:PSS films measured in temperatures ranging from room temperature to $80^{\circ} \mathrm{C}$, and explain the obtained results by proposing the possibility of multiple carrier excitation mechanisms.

\section{Experimental}

The system used to perform the measurements is depicted in Fig. 1. It comprises a PEDOT:PSS film connected to golden electrodes at both ends. A signal generator is used to apply a predetermined sine-wave of $7 \mathrm{~V}$ amplitude and a frequency of $70 \mathrm{~Hz}$ to the sample causing current to flow through the series resistor. The voltage drop across the resistor is recorded and processed to obtain the sample resistance using a personal computer.

To construct the electrodes, a $200 \mathrm{~nm}$ film of gold of $99.9 \%$ purity is deposited via physical vapor deposition (PVD) in $10^{-5}$ torr vacuum on a slide of soda-lime glass. Thermal annealing is performed afterwards for 3 hours at $500{ }^{\circ} \mathrm{C}$. The slides are then cut into $5 \times 12 \mathrm{~mm}$ pieces using $\mathrm{CO}_{2}$ laser. The gold in

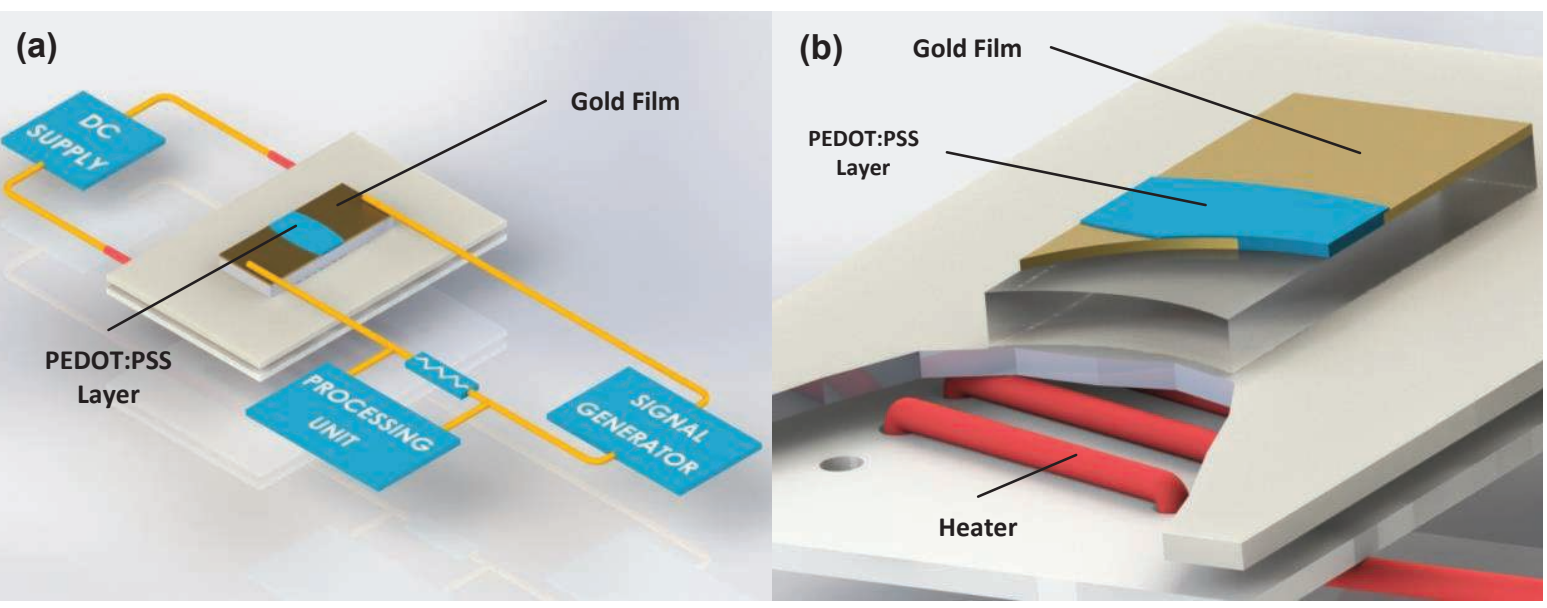

Fig. 1. (a) Schematic diagram of the fabricated device connected to the external measurement circuitry; (b) cross-sectional view of the system. 
a $2 \times 5 \mathrm{~mm}$ area, at the middle of the substrate surface of each chip is scratched away for PEDOT:PSS deposition. The cross-sectional view of the structure is demonstrated in Fig. 1b.

An aqueous solution of PEDOT:PSS prepared using PEDOT:PSS purchased from Sigma-Aldrich is drop casted on the glass slide using a $5 \mu \mathrm{L}$ sampler and left to dry on a heater. A microheater of Al-Fe-Cr wire is constructed underneath the chip allows controlled heating of the sample during the experiments. Prior to each experiment the humidity of the measurement chamber is monitored using a standard hygrometer and adjusted by injection of dry air.

\section{Results}

PEDOT:PSS films of various thicknesses with resistances of $1.8,23$ and $35 \mathrm{M} \Omega$ are tested for temperature dependence of their resistances at two different $\mathrm{RH}$ levels. The samples are heated slowly from room temperature to $80^{\circ} \mathrm{C}$ and the sample's resistance is measured and recorded at equal temperature intervals. The experiment is performed in both an environment of $25 \%$ relative humidity, as well as a dry atmosphere. Results are presented in Fig. 2.

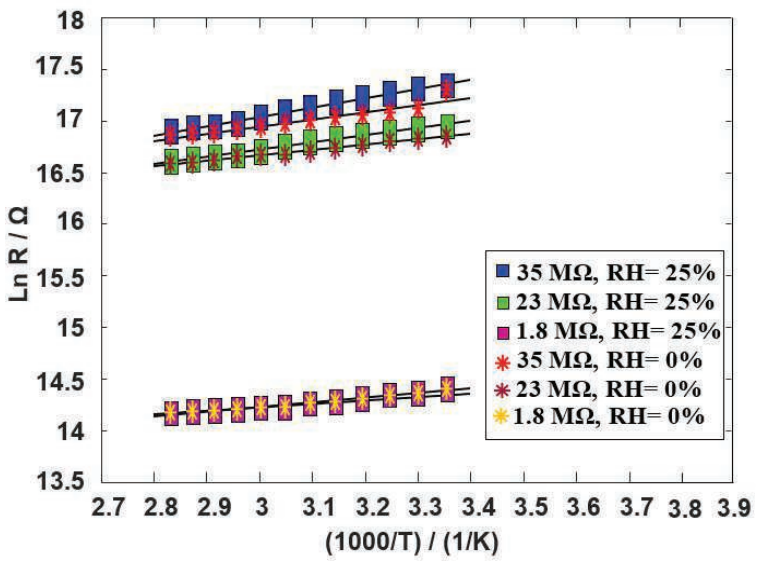

Fig. 2. The change in PEDOT:PSS film resistance with temperature measured in two different $\mathrm{RH}$ levels.

The activation energy in each case is obtained from the slope of the graph, according to Arrhenius's equation:

$$
R=R_{0} \exp \left(\frac{E_{a}}{k T}\right)
$$

Where $R$ is the sample's resistance at the absolute temperature $T, k$ is Boltzmann's constant, and $R_{0}$ is a

Tab. 1: Activation energy for PEDOT:PSS films of different resistances in dry and humid atmospheres

\begin{tabular}{|c|c|c|}
\hline \multirow{2}{*}{$\begin{array}{c}\text { Sample } \\
\text { resistance / } \\
\mathrm{M} \Omega\end{array}$} & \multicolumn{2}{|c|}{ Activation Energy (eV) } \\
\cline { 2 - 3 } & $\mathrm{RH}=0 \%$ & $\mathrm{RH}=25 \%$ \\
\hline 1.8 & 0.03 & 0.04 \\
\hline 23 & 0.04 & 0.06 \\
\hline 35 & 0.06 & 0.08 \\
\hline
\end{tabular}

constant. Table 1 gives the activation energies derived from Fig. 2 for the three samples in both environments.

As can be seen, activation energy decreases with sample thickness. This is because a thicker sample provides more available states for the electric charges to hop to.

Water molecules in air are absorbed by the PEDOT:PSS molecules as demonstrated in Fig. 3, thereby blocking some of the available transport sites for the carriers, which explains the decrease in activation energy with humidity.

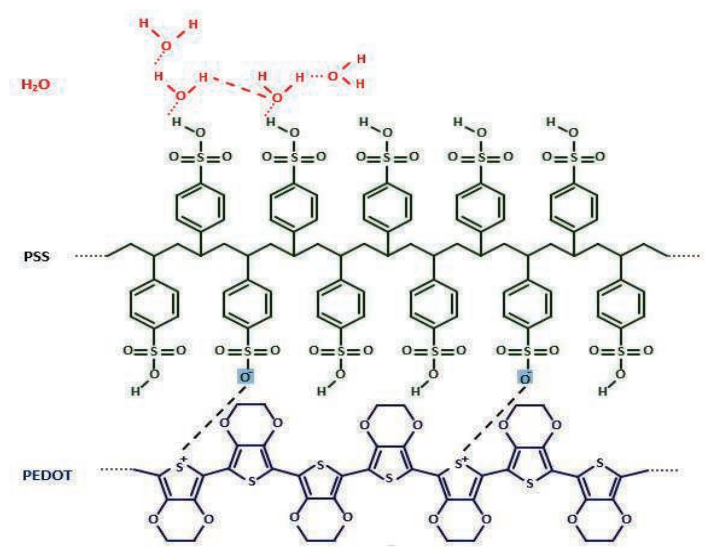

Fig. 3. The molecularl structure of PEDOT:PSS and possible $\mathrm{H}_{2} \mathrm{O}$ absorption sites.

\section{Conclusion}

The conduction activation energy in PEDOT:PSS films decreases with thickness. For a constant thickness, however, the activation energy increases with the $\mathrm{RH}$ at the surrounding atmosphere. The former effect is owing to the higher number of available hopping sites for an electron in a thicker sample, and the latter effect, on the other hand, is due to the site blockage caused by the adsorbed water molecules.

\section{References}

[1] Hossein-Babaei, Faramarz, and Ali Hooshyar Zare. "The selective flow of volatile organic compounds in conductive polymer-coated microchannels." Scientific Reports 7 (2017): 42299. DOI: 10.1038/srep42299

[2] Hossein-Babaei, Faramarz, and Pejman Shabani. "A gold/organic semiconductor diode for ppmlevel humidity sensing." Sensors and Actuators B: Chemical 205 (2014): 143-150. DOI: doi.org/10.1016/j.snb.2014.08.061

[3] Aleshin, A. N., S. R. Williams, and A. J. Heeger. "Transport properties of poly (3, 4ethylenedioxythiophene)/poly (styrenesulfonate)." Synthetic Metals 94.2 (1998): 173-177. DOI: doi.org/10.1016/S0379-6779(97)04167-2 